\title{
Educación y género como variables en la inserción laboral de jóvenes universitarios
}

\section{Education and gender as variables in employment among university students}

\section{ISSN 2071-8748 \\ E-ISSN 2218-3345 \\ (c) (1) (?)}

URI: http://hdl.handle.net/11298/976

DOI: https://doi.org/10.5377/entorno.v0i67.7494
Paola Navarrete Universidad Tecnológica de El Salvador paola.navarrete@utec.edu.sv ORCID: 0000-0001-9280-8389

Recibido: 13 de agosto 2018 Aprobado: 20 de enero 2019

\section{Resumen}

La inserción laboral de los jóvenes en El Salvador es un proceso cambiante y dinámico, el cual responde tanto a las transformaciones de los mercados y las economías globales como a los cambios en la fuerza de trabajo, y a las condiciones socioeconómicas de los individuos. Este artículo explora si el género o el grado académico tienen algún tipo en dicha colocación. De tal forma, el objetivo de la investigación fue determinar la incidencia de variables socioeconómicas en la inserción laboral de los jóvenes salvadoreños. El tipo de estudio fue cuantitativo, para describir y analizar el posicionamiento laboral de los graduados mediante un estudio descriptivo y un código arbitrario de observación (Hernández, Fernández y Baptista, 2014). Se estimó una muestra total de 732 jóvenes salvadoreños, la cual responde a un nivel de confianza estimado del $97 \%$ con un margen de error

\section{Abstract}

The employment of young people in El Salvador is a changing and dynamic process that responds both to changes in markets and global economies as changes in the workforce, as well as the socioeconomic conditions of individuals. This article explores whether gender or academic degree have some influence on that placement. Thus, the aim of the research was to determine the incidence of socioeconomic variables in the employment of young Salvadorans. The type of study was quantitative to describe and analyze the labor positioning of graduates, through a descriptive, exploratory study (Hernandez, Fernandez and Baptist, 2014). A total sample of 732 young Salvadorans was estimated, which corresponds to an estimated level of confidence of $97 \%$ with a margin of error of $4 \%$, and a random distribution and selection. Among the main

\footnotetext{
1 Docente e investigadora de la Facultad de Ciencias Económicas de la Universidad de El Salvador. Coordinadora de la Red de Investigación en diferenciales de Género de Iberoamérica, filial Universidad de El Salvador. Además integran la Red Docentes de la Facultad de Jurisprudencia y Ciencias Sociales, Diana Merino, Multidisciplinaria Oriental, Azucena Retana y el Centro de Estudios de Género, Roselia Núnez.
} 
del $4 \%$, y una distribución y selección al azar. Entre los resultados principales, se tiene que el género no mostró significancia en cuanto a sector de trabajo o salario, pero sí en cuanto a si está trabajando actualmente mientras cursa sus estudios universitarios.

\section{Palabras clave}

Adultos jóvenes-empleo-El Salvador-investigaciones, mercado laboral-El Salvador-investigaciones, búsqueda de empleo, análisis ocupacional, educación y empleo-El Salvador-investigaciones.

\section{Introducción}

Los jóvenes, entre quince a veintinueve años de edad, poseen una mayor probabilidad de encontrarse en situación de desempleo, conformando en el mundo el $35 \%$ de la población total desempleada (Organización Internacional del Trabajo [OIT], 2016).

En El Salvador, los jóvenes conforman el mayor grupo etario, representando el $54 \%$ de toda la población salvadoreña; y al igual que el resto de América Latina, El Salvador demográficamente se ha transformado a tener una población mayormente de jóvenes (Martínez, 2011). A su vez, al ser una población joven, hay más personas en edad y la capacidad de incorporarse y aportar al ámbito económico y laboral.

Pero, a pesar que las tasas en América Latina de desempleos en los jóvenes son elevadas, y que sus características y elementos de inserción laboral son diferentes a la población adulta, existen pocos estudios realizados en El Salvador que den una imagen y una perspectiva que permitan entender las dificultades a las que se enfrentan los jóvenes; que permita entender cómo uno de los grupos poblacionales con mayor potencial no está siendo aprovechado en el sistema económico (Carranza, 2004).

Autores, como Weller (2003), mencionan cómo la dinámica de la inserción laboral de los jóvenes se ve influenciada por las características de los mercados de trabajo latinoamericanos, en donde persisten problemas para la inserción laboral, altas tasas de desempleo y precariedad en el trabajo. Entre algunas de las problemáticas a las results, gender did not show significance in terms of work sector or wage but it did in terms of whether the person is currently working while pursuing university studies.

\section{Keywords}

Young adults-Employment-El Salvador-research, labor Market-El Salvador-research, job search, job analysis, education and Employment-El Salvador-research.

cuales se enfrentan al integrarse al mercado laboral están las siguientes: una débil acumulación de experiencia laboral, que incide negativamente en los ingresos; un desfase entre las características de la educación y las de la demanda laboral tiende a cerrar el canal de la movilidad social; la inserción laboral débil, temprana o tardía, relacionada frecuentemente con altos niveles de deserción escolar, afecta sobre todo a jóvenes procedentes de hogares pobres; jóvenes con inserción laboral precaria son una parte importante de la población de riesgo con problemas de adaptación y marginación social (Weller, 2003).

Comprender los procesos de inserción laboral, y las características de los primeros empleos en la población joven, nos permite entender los comportamientos y las maneras en las cuales los jóvenes logran la inserción laboral. Que a su vez permite identificar las desigualdades que actualmente deben de enfrentar los jóvenes en el mercado laboral (García-Blanco \& Gutiérrez, 1996)

\section{Inserción al mundo Laboral}

Para García-Blanco y Gutiérrez (1996) inserción laboral es el término utilizado habitualmente para referirse al proceso de incorporación a la actividad económica de los individuos, que suele coincidir, para la mayoría de los miembros en la sociedad, con la etapa juvenil, constituyéndose como un proceso de transición social desde el sistema educativo y de la familia de origen hacia posiciones del mercado de trabajo y de la independencia familiar.

Podemos definir a la inclusión o inserción laboral "como el acceso a puestos de trabajo productivos, adecuadamente 
renumerados y con condiciones dignas" (Comisión Económica para América Latina y el Caribe/ Organización Iberoamericana de Juventud [CEPAL/OIJ], 2003). La importancia de la inserción de los jóvenes en el mundo laboral no solamente radica en que puedan establecerse en un trabajo; ello también implica que sea un trabajo digno. Trabajar es una parte importante para el desarrollo de los individuos en una sociedad; no solo permite que las personas se ganen su sustento y tener seguridad económica, sino además que los ciudadanos participen plenamente en la sociedad, confiriéndoles un sentido de dignidad y valía personal. El trabajo se transforma en un elemento vital para lograr un crecimiento económico equitativo, logrando la reducción de la pobreza y desigualdad de género (PNUD, 2015).

\section{Educación superior y trabajo en jóvenes}

La decisión de los jóvenes de participar o no participar en el mercado de trabajo es una cuestión compleja que depende de varios factores económicos y sociales. Cada uno de estos factores puede impulsar al individuo a cursar estudios profesionales 0 a decidirse participar en el mercado de trabajo (OIT, 2016a).

La oferta laboral también influye en la disposición de los jóvenes de seguir cursando estudios y en no buscar empleo. Por ejemplo, un incremento de las oportunidades de empleo en ocupaciones que requieren de individuos altamente calificados podría incitar a los jóvenes a seguir cursando estudios. A su vez, ofrecer mayores salarios por empleos que no requieren de calificaciones puede disuadirlo a seguir estudiando (OIT, 2016).

Hoy en día los jóvenes latinoamericanos tienen un mayor nivel de educación formal que la que tuvieron las generaciones pasadas, pero esto no garantiza la inserción al mundo laboral. Para Weller (2006), este fenómeno es dado que la demanda laboral está sesgada hacia la búsqueda de personal más calificado.

La educación generalmente es vista como un elemento central para la mejora en la inserción laboral de los jóvenes, existiendo la noción de que a mayor nivel de educación se reduce el riesgo a ser desempleado (Diez de Medina 2001; citado en Weller, 2003). Pero actualmente existe el debate si realmente la educación es un vehículo que permite mejorar el proceso de inserción laboral.
Para Duryea y Pagés (2002; citado en Weller, 2003), una mejor educación en muchos casos tiene un impacto limitado en la mejoría de los ingresos; no existiendo una garantía de mayores oportunidades de empleo por haber obtenido un título de mayor nivel educativo.

La calidad de la educación es otro de los factores que afecta a la hora de insertarse, a pesar de que, en América Latina, varios países poseen educación primaria y secundaria con cobertura nacional. En ocasiones va de la mano con mediciones mediocres de los resultados, segmentación de sistemas educativos, devaluaciones educativas y problemas de cobertura (Weller, 2006); no garantizando que el acceso a estos sistemas educativos propicie una formación de calidad.

Enjóvenes de 15a 19 años de edad, que no están participando activamente en el mundo laboral, generalmente se puede interpretar como un avance económico y social positivo, pues implica que buena parte de los adolescentes y jóvenes no necesitan trabajar para generar ingresos, permitiendo que puedan seguir desarrollando sus competencias y aumentando su nivel educativo con miras a tener mejores oportunidades de empleo en el futuro. sin embargo, la tasa de participación en la mano de obra de los jóvenes, en este rango de edad, varía considerablemente de una región a otra, lo cual pone de manifiesto varios problemas y oportunidades (OIT, 2016a).

Es en la educación superior donde se produce un salto significativo en los ingresos, y con ello mayores oportunidades de bienestar. La familia de origen de los jóvenes y su formación educativa generan un impacto significativo en los ingresos del hogar y futuros ingresos de los jóvenes. Ganando un hijo o nieto de un profesional casi un $36 \%$ más que otro joven, hijo o nieto, de un trabajador no calificado (Ibáñez Schuda, 2002).

Otro aspecto que forma parte de los obstáculos identificados, que tienen los jóvenes al insertarse, son los perfiles laborales demandados por las empresas, que en muchos casos solicitan una vasta experiencia laboral, un alto nivel académico (con especialización en algunos casos) y una edad relativamente joven (típicamente entre 25 y 30 años), dificultando el acceso de los jóvenes a empleos decentes y altamente productivos (OIT, 2012). 
Jóvenes que estudian y trabajan

Para Handal (2014), los jóvenes de entre 20 y 24 años que se encuentran inactivos por motivos de estudio tratan de iniciar su ingreso al mercado laboral como trabajadores temporales o bajo la figura de no remuneración. Usualmente con el objetivo de ganar la experiencia necesaria para obtener un mejor empleo. Pero la combinación de estudios y trabajo en forma adecuada puede ayudar a jóvenes a desarrollar estrategias laborales individuales y generar la autoestima para tomar sus primeros pasos; exceptuando en jornadas laborales extensas o extenuantes, el trabajo afectará el rendimiento escolar, restringiendo la capacidad de aprendizaje y aumentando una tendencia negativa (Weller, 2006).

Pero trabajar proporciona una relevante experiencia para la inserción laboral. Trabajar durante la asistencia al sistema escolar, en una manera que no afecta significativamente el rendimiento de los estudios, no solo generaría ingresos, sino que los acercaría al mundo laboral al poder desarrollar y poner en práctica destrezas que no son relevantes en el ámbito escolar (Krauskopf, 2003).

\section{Brechas de género}

Brechas en el mercado de trabajo afectan no solo a los individuos, sino que además constituyen obstáculos para el crecimiento y la reducción de la desigualdad y la pobreza (OIT, 2016b). Weller (2006) manifiesta que, ahora más que nunca, las mujeres jóvenes valoran cada vez más realizar trabajos fuera del hogar, considerándose un elemento clave para el desarrollo de la autoestima y sus perspectivas de vida, convirtiéndose el empleo en una manera de independizarse de los papeles tradicionales de las mujeres vinculados con el hogar.

Pero particularmente las mujeres se enfrentan con distintas barreras sociales, cultures o políticas, además de los factores económicos que influyen en su decisión para entrar al mercado laboral o seguir cursando sus estudios (OIT, 2016a). Para Weller (2006), las mujeres enfrentan problemas particulares a la hora de insertarse en el ámbito laboral, siendo notorias las dificultades que tienen en su formación educativa, normalmente baja; para las cuales hay muy pocas oportunidades de empleo productivo. Las mujeres también muestran brechas en las tasas de desempleo con sus coetáneos masculinos, disminuyéndose en grupos educativos más altos.
En un estudio elaborado por la OIT (2016), se encontró que existen las mismas causas del porqué hay inactividad entre distintos grupos de mujeres trabajadoras. Para la mayoría de los encuestados, esta inactividad se debe a las responsabilidades familiares, al trabajo doméstico y a la falta de ganas de trabajar. Pero siguen siendo el matrimonio y la maternidad prematura los principales factores limitantes al acceso de las mujeres a la integración al mercado de trabajo y a la educación (Elder y Kring, 2016; citando en OIT, 2016). Debido a esto, de igual forma tienen las mujeres jóvenes mayores probabilidades de lograr empleos informales y vulnerables, ya que son más propensas a ser trabajadoras familiares no remuneradas (OIT, 2016b).

\section{Método}

\section{Diseño y tipo de estudio}

Se implementó una metodología cuantitativa para describir y analizar el posicionamiento laboral de los jóvenes mediante un estudio descriptivo, exploratorio, utilizando un código arbitrario de observación (Hernández, Fernández y Baptista, 2014).

Instrumentos por utilizar para la recolección de datos

Para la recolección de la información, se utilizaron encuestas estructuradas de tipo cerrado (Hernández, Fernández y Baptista, 2014), conformadas por ítems de nivel nominal dicotómico, polinómico y de respuesta variable cerrada. La información recolectada se almacenó en una base de datos para su análisis en el programa IBM SPSS Statistics v.19.

\section{Universo y muestra}

De acuerdo con los datos de la Encuesta de Hogares de Propósitos Múltiples del año 2016 (2017), hay en el país 895.161 jóvenes entre los 15 y los 29 años de edad que han estudiado diez o más años, equivalentes a estudios de bachillerato y de nivel universitario.

Con la población de jóvenes antes mencionada, se estimó una muestra total de 732 participantes para el estudio, con base en la fórmula para el cálculo de poblaciones finitas, la cual responde a un nivel de confianza estimado del $97 \%$ y un margen de error del $4 \%$, con distribución y selección al azar. 


\section{Descripción de la población encuestada}

En cuanto a los datos sociodemográficos de la población sondeada (ver tabla 1), 268 de los encuestados son del sexo masculino (36,5\% de la muestra), mientras que $461(62,7 \%)$ corresponden al femenino. Según su edad, aquellos jóvenes entre los 18 y los 20 años conforman en la muestra el $47,9 \%(n=352)$, mientras que los jóvenes de 21 años hasta los 25 representan el $29 \%(n=213)$, siendo por último los rangos de 26 a 30 años correspondientes al $15,5 \%(n=114)$ de la población sondeada.

Tabla 1.

Características sociodemográficas de los participantes

\begin{tabular}{|c|c|c|c|c|}
\hline & Variables & Frecuencia & Porcentaje & Porcentaje válido \\
\hline \multirow[t]{3}{*}{ Sexo } & Masculino & 268 & 36,5 & 36,8 \\
\hline & Femenino & 461 & 62,7 & 63,2 \\
\hline & NS/NR & 6 & 0,8 & \\
\hline \multirow[t]{4}{*}{ Edad por rango } & 18 a 20 & 352 & 47,9 & 51,8 \\
\hline & 21 a 25 & 213 & 29,0 & 31,4 \\
\hline & 26 a 30 & 114 & 15,5 & 16,8 \\
\hline & $\mathrm{Ns} / \mathrm{Nr}$ & 56 & 7,6 & \\
\hline \multirow[t]{15}{*}{ Departamento } & Ahuachapán & 44 & 6,0 & 6,0 \\
\hline & Santa Ana & 41 & 5,6 & 5,6 \\
\hline & Sonsonate & 49 & 6,7 & 6,7 \\
\hline & La Libertad & 84 & 11,4 & 11.4 \\
\hline & Chalatenango & 46 & 6,3 & 6,3 \\
\hline & Cuscatlán & 34 & 4,6 & 4,6 \\
\hline & San Salvador & 213 & 29,0 & 29,0 \\
\hline & La Paz & 44 & 6,0 & 6,0 \\
\hline & Cabañas & 35 & 4,8 & 4,8 \\
\hline & San Vicente & 37 & 5,0 & 5,0 \\
\hline & Usulután & 31 & 4,2 & 4.2 \\
\hline & San Miguel & 23 & 3,1 & 3,1 \\
\hline & Morazán & 24 & 3,3 & 3,3 \\
\hline & La Unión & 30 & 4,1 & 4,1 \\
\hline & Total & 735 & 100,0 & 100,0 \\
\hline
\end{tabular}

\section{Resultados}

\section{Caracterización de la población que ha trabajado}

Al contrastarse con la variable Ha trabajado con la variable Sexo, los hombres muestran una mayor frecuencia de haber desempeñado un trabajo, con un $76,7 \%(n=197)$ en comparación con las mujeres (50,8 \%), siendo una minoría los jóvenes que nunca han realizado trabajo, un $23,3 \%(n=60 \%)$, pero que es más notorio en las mujeres, con $49,2 \%(n=219)$. A nivel general de la muestra, eran $60,2 \%(n=426)$ los que manifestaron haber trabajado. 
Tabla 2.

Tabla de contingencia de la variable Ha trabajado con la variable Sexo

\begin{tabular}{|c|c|c|c|c|c|c|c|}
\hline \multirow[t]{2}{*}{ Masculino } & & \multicolumn{4}{|c|}{ 1. Sexo } & \multirow[b]{2}{*}{ Total } & \multirow{2}{*}{$\begin{array}{c}\text { Pruebas de } \\
\text { Chi-cuadrado de } \\
\text { Pearson }\end{array}$} \\
\hline & & $\begin{array}{c}\% \text { del } n \text { válido } \\
\text { de columna de } \\
\text { capa }\end{array}$ & Femenino & $\begin{array}{c}\% \text { del } N \text { válido } \\
\text { de columna de } \\
\text { capa }\end{array}$ & & & \\
\hline \multirow[t]{2}{*}{ 4. Ha trabajado } & Sí & 197 & 76,7 & 226 & 50,8 & & $X^{2}: 45.518$ \\
\hline & No & 60 & 23,3 & 219 & 49,2 & & gl: 1 \\
\hline Total & & 257 & 100,0 & 445 & 100,0 & 702 & Sig. .000 \\
\hline
\end{tabular}

Estos valores mostraron un nivel de significancia, según la prueba de Chi-cuadrado de Pearson de $\chi^{2}=0.000$, indicando una significancia estadística entre las variables. En cambio, al preguntarles si actualmente se encontraban laborando, únicamente un $35,0 \%(n=245)$ de la muestra general manifestó estarlo haciendo. Al comprar entre los géneros, podemos ver una diferencia entre ellos. En el caso de los hombres, el 45,7\% $(n=118)$ está actualmente trabajando, mientras que solo el $29,1 \%$ $(n=127)$ de las mujeres está trabajando actualmente.

Tabla 3.

Variable Se encuentra trabajando en contraste con la variable Sexo

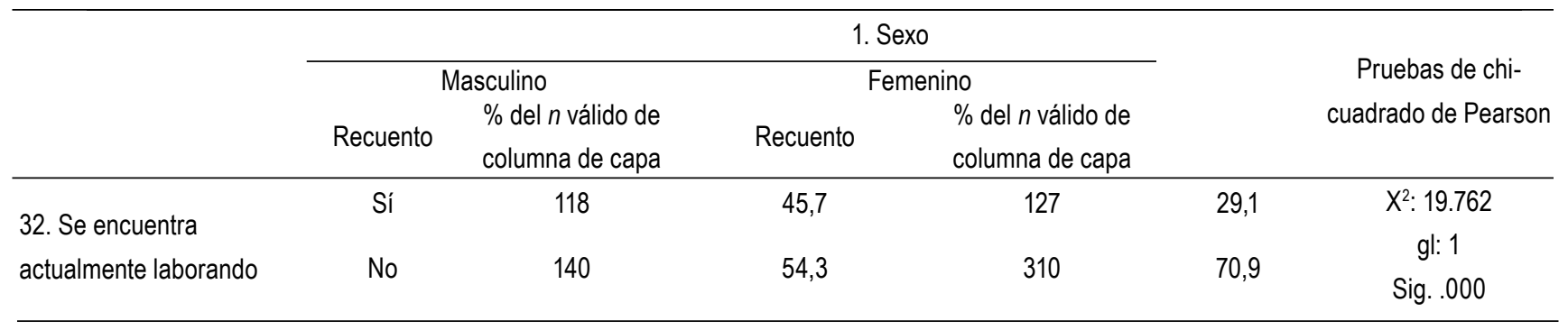

De la población que se encuentra trabajando, se tiene un promedio salarial de $\$ 455.5$, en el caso de los hombres, y de $\$ 404.8$, en el de las mujeres. No se encontró nivel de significancia en dichas cifras.

\section{Perfil actividad económica en jóvenes}

Al comparar las características económicas de los jóvenes, no se pueden distinguir cambios significantes entre estatus laboral, sector o actividades económicas entre los participantes. Tanto mujeres como hombres presentan tener, por lo general, un estatus laboral de contratación definitiva o permanente, siendo $61,2 \%$, en el caso de los hombres, y de $59,2 \%$, en el de las mujeres. Según el sector de trabajo, ambos están ejerciendo en el sector privado; pero hay un porcentaje levemente más alto en el caso de mujeres que trabajan con su familia, como también trabajan más en el sector informal $(12,0 \%$ en comparación con el $9,6 \%$ en el caso de los hombres). 
Tabla 4.

Características de la actividad económica

\begin{tabular}{|c|c|c|c|c|c|c|}
\hline & & & & 1. Sexo & & \\
\hline & & ulino & & Femenino & & \\
\hline & Recuento & $\begin{array}{l}\% \text { del } n \text { válido de } \\
\text { columna de capa }\end{array}$ & Recuento & $\begin{array}{c}\% \text { del } n \text { válido de columna } \\
\text { de capa }\end{array}$ & $\begin{array}{c}\text { Pruebas de Chi- } \\
\text { cuadrado de } \\
\text { Pearson } \\
\end{array}$ & \\
\hline & Contratación & & & & & \\
\hline & definitiva & 71 & 61,2 & 74 & 59,2 & \\
\hline $\begin{array}{l}\text { 35. Estatus de la } \\
\text { relación laboral }\end{array}$ & $\begin{array}{c}\text { Contratación } \\
\text { temporal }\end{array}$ & 27 & 23,3 & 26 & 20,8 & $\begin{array}{c}X^{2}: 9.603 \\
\text { gl: } 8\end{array}$ \\
\hline & Negocio propio & 8 & 6,9 & 11 & 8,8 & Sig. .294 \\
\hline & Otros & 10 & 8,6 & 14 & 11,2 & \\
\hline & Público & 21 & 19,4 & 33 & 27,3 & \\
\hline 36. Sector de donde & Privado & 68 & 63,0 & 71 & 58,7 & $X^{2}: 6.405$ \\
\hline $\begin{array}{l}\text { está laborando se } \\
\text { considera }\end{array}$ & Familiar & 10 & 9,3 & 13 & 10,7 & $\begin{array}{c}\text { gl: } 4 \\
\text { Sig. . } 171\end{array}$ \\
\hline & Mixto & 9 & 8,3 & 3 & 2,5 & \\
\hline 37. Sector & Formal & 85 & 90,4 & 95 & 88,0 & $X^{2}: .314$ \\
\hline & Informal & 9 & 9,6 & 13 & 12,0 & Sig. .575 \\
\hline & Servicios & 51 & 50,0 & 56 & 51,4 & \\
\hline & industriales, & 8 & 7,8 & 16 & 14,7 & $X^{2}: 8.863$ \\
\hline 38. Acilvidad & comerciales, & 27 & 26,5 & 17 & 15,6 & gl: 8 \\
\hline & otros & 16 & 15,7 & 20 & 18,3 & Sig. 354 \\
\hline & No & 3 & 2,8 & 5 & 4,3 & \\
\hline
\end{tabular}

En cuanto a la actividad económica, en ambos casos hay un mayor porcentaje de participantes que trabajan en el sector de servicios; pero existe una mayor cantidad de mujeres trabajando en el sector industrial en contraste con los hombres, que tienen una mayor cantidad en el sector comercial.

En el caso de los jóvenes que no se encuentran trabajando, al preguntarles cuáles son las razones, en la muestra total el $69,0 \%(n=292)$ manifestó que era debido a que estaba estudiando, mientras que un $21,7 \%(n=92)$ manifestó que no estaba buscando o que no encuentra trabajo. Al compararlo con el sexo, los hombres manifestaron, en un $67,7 \%$, que era debido a sus estudios; $y$ en el caso de las mujeres, en un 70,0 \% es debido a este motivo. De esta población inactiva, un $48,7 \%$ de los hombres han buscado trabajo activamente, y en el caso de las mujeres, un $41,2 \%$. 
Tabla 5.

Características de jóvenes inactivos

\begin{tabular}{|c|c|c|c|c|c|c|}
\hline & \multicolumn{5}{|c|}{ 1. Sexo } & \multirow{3}{*}{$\begin{array}{l}\text { Pruebas de Chi- } \\
\text { cuadrado de Pearson }\end{array}$} \\
\hline & \multicolumn{2}{|c|}{ Masculino } & \multicolumn{2}{|c|}{ Femenino } & & \\
\hline & Recuento & $\begin{array}{l}\% \text { del } n \text { válido de } \\
\text { columna de capa }\end{array}$ & Recuento & $\begin{array}{l}\% \text { del } n \text { válido de } \\
\text { columna de capa }\end{array}$ & & \\
\hline \multirow{3}{*}{$\begin{array}{l}\text { 47. Ha buscado trabajo en } \\
\text { los últimos } 12 \text { meses }\end{array}$} & Sí & 58 & 48,7 & 113 & 41,2 & $\begin{array}{l}\mathrm{X}^{2}: 1.898 \\
\mathrm{gl}:\end{array}$ \\
\hline & $\begin{array}{c}\text { No } \\
\text { Trabajo doméstico }\end{array}$ & 61 & 51,3 & 161 & 58,8 & Sig. .168 \\
\hline & $\begin{array}{l}\text { / obligaciones } \\
\text { personales }\end{array}$ & 1 & 0,8 & 10 & 3,4 & \\
\hline \multirow{4}{*}{$\begin{array}{l}\text { 46. Si no se encuentra } \\
\text { laborando, por qué motivos } \\
\text { no está laborando }\end{array}$} & Estudia & 88 & 67,7 & 203 & 70,0 & $X^{2}: 16.528$ \\
\hline & Está buscando & & & & & gl: 11 \\
\hline & $\begin{array}{l}\text { trabajo / No } \\
\text { encuentra trabajo }\end{array}$ & 30 & 23,1 & 60 & 20,1 & Sig. .123 \\
\hline & Otros & 11 & 8,5 & 17 & 5,9 & \\
\hline
\end{tabular}

\section{Conclusiones}

Las variables socioeconómicas que inciden en la obtención de un empleo son varias, rondando desde la educación formal hasta variables de carácter subjetivo, como las recomendaciones al interior de la empresa.

Al indagar si existe relación entre el sexo y la inserción laboral, no se encontraron datos que fuesen estadísticamente significativos a ese respecto. Sin embargo, sí se ven diferencias por sexo en cuanto a la cantidad de ingresos, donde los hombres siguen ganando más que las mujeres por las mismas actividades realizadas, lo cual es percibido como un indicador de desigualdad por los participantes del estudio. Las diferencias por sexo no se muestran en los otros contrastes. Trabajar en una empresa pública, privada o familiar no depende de ello, ni ninguna de las otras variables asociadas con dicho indicador.

Asimismo, la mayoría de participantes estuvo de acuerdo con que en su trabajo existe la disposición de dejarlos estudiar y perseguir una carrera de educación superior, pero que el tiempo y los recursos para ello dependen del mismo estudiante, es decir, se percibe una cultura de que se permite, mas no se facilita. Pese a esto, la mayoría de participantes que no se encuentran estudiando estarían dispuestos a estudiar en un horario laboral accesible para sus turnos de trabajo.

En cuanto a la relación entre estar laborando actualmente y el nivel educativo, se observó que sí es un factor determinante en la obtención de un empleo, particularmente en los sectores de industria, comercio y servicios, donde ser bachiller es mandatorio en la mayoría de los casos; y la población, subsecuentemente, consigue dicho grado académico. De tal forma, se puede decir que, según la configuración actual de los requerimientos de empleo formal, el nivel educativo sí es relevante.

A pesar de que demográficamente existe una mayor cantidad de mujeres que de hombres en El Salvador, es menor la cantidad de mujeres que están activamente participando en el mundo laboral, esto también se puede ver en las tasas de inactividad y la tasa global de participación. 


\section{Referencias}

Carranza, M. (2005). "Inserción de las y los jóvenes en el mercado laboral salvadoreño". Estudios Centroamericanos. Recuperado de https://scholar. google.es/scholar?hl=es\&as_sdt=0\%2C5\&q=Inserci\% $\mathrm{C} 3 \% \mathrm{~B} 3 \mathrm{n}+\mathrm{de}+\mathrm{las}+\mathrm{y}+\mathrm{los}+\mathrm{j} \% \mathrm{C} 3 \% \mathrm{~B} 3 \mathrm{venes}+\mathrm{en}+\mathrm{el}+$ merca do+laboral+salvadore\%C3\%B10.+Estudios+Centroam ericasnos\&btnG=

Comisión Económica para América Latina y el Caribe. Organización Iberoamericana de Juventud (2003). "Juventud e inclusión social en Iberoamérica". Recuperado de https://repositorio.cepal.org/ bitstream/handle/11362/31842/S2003692_ es.pdf? sequence $=1$ \&isAllowed $=\mathrm{y}$

Equipo técnico de trabajo decente de la OIT para América Central, Haití, Panamá y República Dominicana (2011). "Perspectiva laboral El Salvador". En G.E. Martínez (Ed.). “Empleo y emprendimiento juvenil:desafíos para el crecimiento económico y la integración social". pp. 15-41. Recuperado de https://www.ilo.org/wcmsp5/ groups/public/---americas/---ro-lima/---sro-san_jose/ documents/publication/wcms_185735.pdf

García-Blanco, J.M., \& Gutiérrez, R. (1996). "Inserción laboral y desigualdad en el mercado de trabajo: cuestiones teóricas". Revista Española de Investigaciones Sociológicas. 75, pp. 269-293.

Handal, G. (2014). "Transiciones en el mercado de trabajo de las mujeres y hombres jóvenes en El Salvador". Work4Youth. Serie de publicaciones. Recuperado de https://www.ilo.org/wcmsp5/groups/public/@ dgreports/@dcomm/documents/publication/ wcms_326253.pdf
Ibáñez Schuda, S.A. (2002). "Estudio de caso N. 64. Inserción laboral juvenil: análisis de variables relevantes y perspectivas de política". Recuperado de http:// www2.congreso.gob.pe/sicr/cendocbib/con4_uibd. nsf/A01A21483B2CD72705257DB70062D675/\$FI LE/200711200019_4_2_0.pdf

Organización Internacional del Trabajo (2012). "Diagnóstico del empleo juvenil en El Salvador 2012". Recuperado de https://www.ilo.org/wcmsp5/groups/public/---ed_ emp/---ed_emp_msu/documents/genericdocument/ wcms_231593.pdf

Organización Internacional del Trabajo. (2016). “Perspectivas Sociales y del Empleo en el Mundo 2016. Tendencias entre los jóvenes". Ginebra: Organización Internacional del Trabajo. Recuperado de http://www.ilo.org/global/ research/global-reports/youth/2016/WCMS_513747/ lang--es/index.htm

Programa de las Nacionas Unidas para el Desarrollo (2015). "Informe sobre desarrollo humano 2015. Trabajo al servicio del desarrollo humano". Recuperado de http:// hdr.undp.org/sites/default/files/hdr_2015_overview_ sp_final.pdf

Weller, J. (2003). "Problemas de la inserción laboral de la población juvenil en América Latina". Papeles de población. Recuperado de https://www.redalyc.org/ articulo.oa?id=11204901

Weller, J. (2006). "Inserción laboral de jóvenes: expectativas, demanda laboral y trayectorias". Recuperado de https://scholar.google.es/scholar?hl=es\&as_ sdt=0\%2C5\&q=Inserci \%C3\%B3n+laboral+de+j\%C3\% B3venes\%3A+expectativas\%2C+demanda+laboral+y+ trayectorias\&btnG $=$ 